\title{
A High-Resolution Networked Computer System for Radiologic Instruction of Medical Students
}

\author{
David B. Hayt, R. James, R. Knowles, and Steven M. Erde
}

\begin{abstract}
A network of 18 Macintosh I1-based workstations (Apple Computer Corp, Cupertino, CA) having highresolution 1,024-line monitors is used for the radiologic instruction of medical students in our institution. Although this is an expensive system, it is time-shared with the Departments of Pathology, Physiology, Neuroscience, Biochemistry, Parasitology, and Microanatomy. The number of workstations available allows the entire class to access the system simultaneously by working in small groups. Because of its high resolution, medical imaging studies and text may be displayed on the same monitor side by side. We have chosen a question-and-answer format (Carousel). Forty-one radiologists in our affiliated medical institutions contributed cases. These cases in question-andanswer form supplement the lectures and film-based tutorials given as part of our mandatory third year radiology clerkship.

Copyright $(1991$ by W.B. Saunders Company
\end{abstract}

KEY WORDS: computers, teaching, networks.

$\mathbf{C}^{\circ}$ OMPUTER-ASSISTED teaching of medical students has become increasingly common in our institution. A 1,024-line, 8-bit depth, high-resolution color system developed by the Department of Pathology is now being used in multiple locations within our medical school for medical student instruction in a variety of subjects. The high-resolution capabilities of this system, in addition to its other attributes, make it ideal for instruction of medical students in their first clinical rotation, radiology, at the beginning of the third year. Although the system is not inexpensive, its large capacity and networked multiple stations make it applicable to many areas of medical student teaching, thereby distributing its cost over many departments and subjects.

\section{MATERIALS AND METHODS}

Radiologic medical student instruction (called RAD$\mathrm{MAC}$ ) is one of the courses available through the PathMAC

From the Departments of Radiology and Pathology, The New York Hospital-Comell University Medical College, New York, NY.

Address reprint requests to David B. Hayt, MD, Associate Professor of Radiology, The New York Hospital-Comell Medical Center, 525 68th St, St-8a33, New York, NY 10021.

Copyright (1) 1991 by W.B. Saunders Company

0897-1889/91/0404-0003\$03.00/0
Computer Laboratory developed by the Department of Pathology. PathMAC consists of 18 Macintosh II (Apple Computer Corp) workstations linked by Ethernet. There are clusters of workstations available in the Department of Pathology and in the two student residences. This network currently spans three buildings, and is linked to the growing fiberoptic/twisted pair Ethernet backbone in the hospital.

Each workstation consists of a Macintosh II with 5 Mbyte of random access memory (RAM), 20-megabyte hard disk, Ethernet card, and a 19-inch 1,024 × 780 line highresolution 8-bit color Supermac display. The system uses multiple AppleShare file servers with over 2.5 gigabytes of hard disc space for central storage of text and digitized images. Images are digitized from $35-\mathrm{mm}$ slides in a $1,500 \times$ 1,000 pixel format, at 24-bit depth for color, and 8 bits for black and white with a Barneyscan slide scanner. They are then cropped, sharpened, and resized. A Sharp JX-450 (Sharp Electronics Corp, Mahwan, NJ) flatbed scanner is used for prints and figures. Because color is required for gross and microscopic images, the 24-bit image is compressed to a custom palette (color look-up table) to assure the proper display colors at the workstation and to decrease image size. The 8-bit images are one third the size of the 24-bit images and average between $100 \mathrm{~K}$ and $200 \mathrm{~K}$ bytes. Images can be retrieved from the file server over the Ethernet at a rate of up to $200 \mathrm{~K}$ bytes/s, which translates to a retrieval time of less than 2 seconds per image.

Hyperpath is a multimedia Hypertext instruction system for the pathophysiology course based on Guide (version 2.0, OWL International, Bellvue, WA). Guide allows the full integration of text and images ranging from pathology slides to radiologic studies. Students can navigate completely individualized paths through the material by using a mouse and Macintosh graphic interface. For example, students can move from the term splenomegaly to a definition, clinical signs and symptoms, gross and microscopic images, nuclear medicine and computed tomography images, and back, all with the click of a mouse. (A somewhat different approach to hypertext/hypermedia was presented by Jaffe et al. ${ }^{1}$ )

For the third-year radiology course, material is organized in carousels. Carousels are an application developed at our institution to provide a linear review mechanism for the students that emulates a slide project. It was designed to use a simple format: present an image; ask a question, and then provide the answer with reinforcement. This requires minimum work by the instructor to create an effective presentation. (For the value of immediate reinforcement, see Aronberg et al. $\left.^{2}\right)$

Medical students can use the workstations at their convenience 24 hours a day. After activating a station, a medical student uses a mouse to open the radiology folder. Inside the folder, the student selects from various modules. Each module is composed of one or two carousels of images with accompanying text. Each module is related to a specific area of radiology (Fig 1). The medical student is directed to 
Fig 1. Three windows that the student sees upon entering the computer teaching system. The first window in the upper left is labeled PathMAC II. This is the root directory of the file server, which graphically shows the multiple courses available as individual folders. When a student opens a folder, as in the window titled "Radiology" in the upper right, he sees the multiple subchoices for that particular course. If one of these folders is subsequently opened, as in the lower left, "Abdominal Gas Patterns," the student can see that there are two modules available, called carousels.

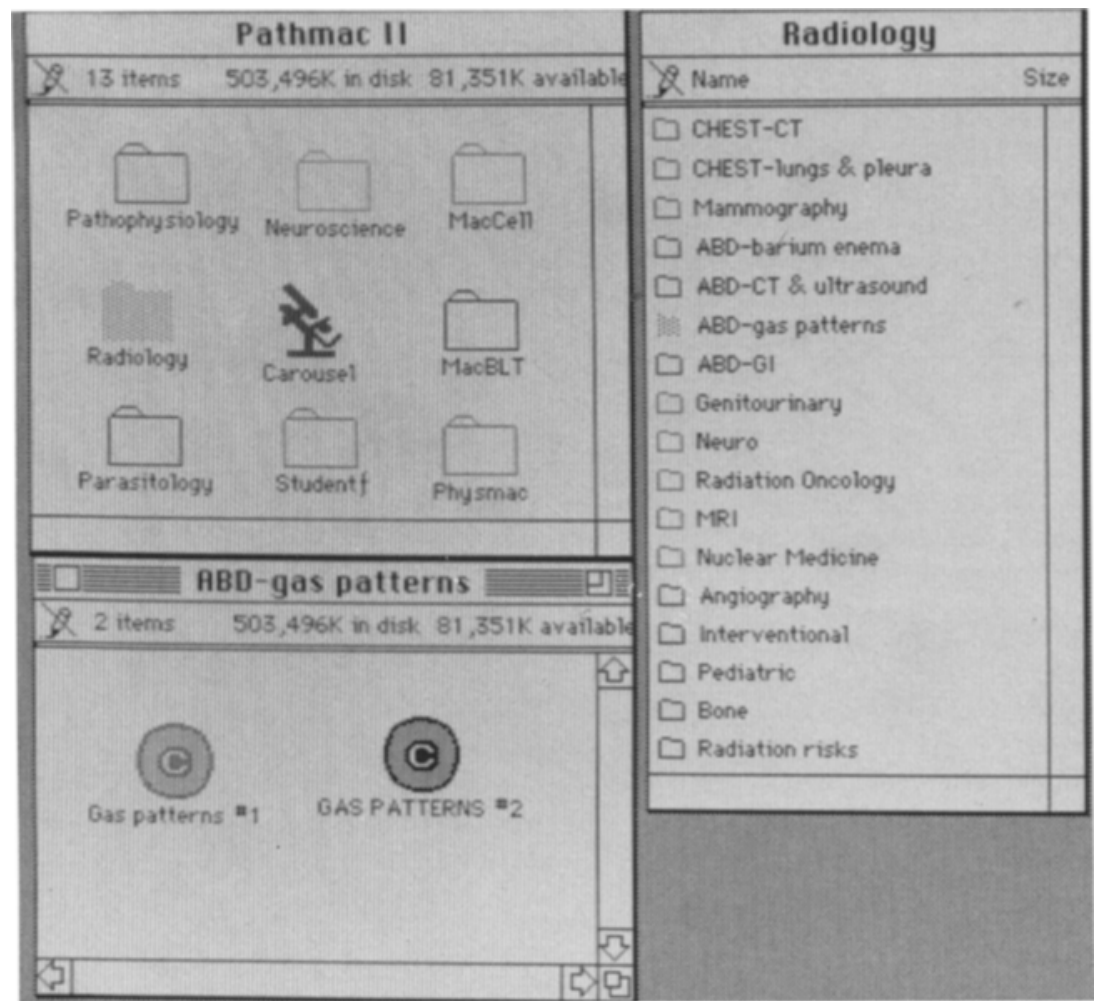

answer various questions concerning individual images or image sets. He or she may jump backward or forward, and may search for images by keywords. Images may be enlarged, or more than one image may be viewed at a time. Radiologic images may be labeled or outlined in color, or may have colored arrows added (Fig 2). Local areas on the image may be lightened or darkened; the contrast may be changed; variable degrees of edge enhancement may be added through a variety of filters-these changes may be blended with the remainder of the image through an

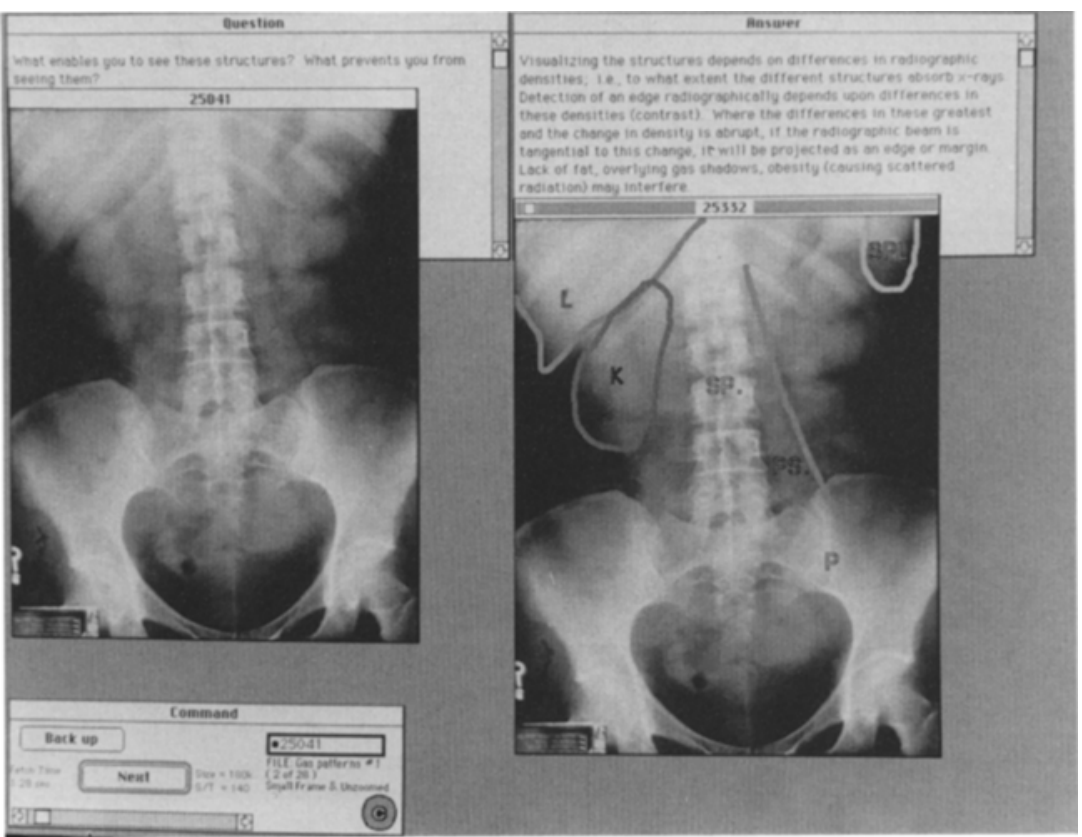

Fig 2. Carousel 1: Abdominal Gas Patterns. The screen shows the question-and-answer windows as well as images with and without colored overlays related to the questions and answers of this particular module. 
adjustable width-blending program. This enables optimum quality image presentation. Even if only $25 \%$ of the screen area is used for a radiologic image, the 525 -line reproduction will be superior to a standard video image.

Most carousels use the left hand side of the screen for images and the right hand side for a sequential question and answer text (Fig 3). Because of the high resolution of the screen, fairly extensive explanations of the radiologic findings may be given in the answer without scrolling the text (Fig 4).

For the initial images and text, 41 radiologist subspecialists in the affiliated hospitals of the medical school system contributed five cases each, with an average of five questions and answers per case in their area of expertise. These were incorporated into carousels, one carousel per radiologist, and all carousels in a subspecialty area grouped into a module (ie, neuroradiology, etc).

After editing, adding appropriate arrows, changing text, and making other minor revisions, each case was reassigned to a single mandatory (or, in some cases, an additional optional) carousel per module. Cases are arranged in each module depending on the difficulty of the case and the subject. Difficult cases are assigned to optional carousels to permit programmed learning. ${ }^{3}$ Course objectives mandate that the student be able to interpret chest radiographs, plain films of the abdomen, and fracture films, and that he must be familiar with all other areas of medical imaging regarding the capabilities, advantages and disadvantages, and image choices available for selected disease processes. ${ }^{4}$ Therefore, all carousels in chest, plain films of the abdomen, and fracture modules are mandatory.

Case material was selected to parallel the lecture series that are part of the course. This reinforces but does not duplicate either the lecture material or the lecture slides to provide cluster learning. ${ }^{3}$
The unedited carousels were initially compared with commercially available medical student-level slide-tape lectures, using 12 third-year medical students who were participating in the mandatory third-year radiology clerkship, in May 1989, near the end of their third year. (Subsequent to this group, the new course commenced at the end of June and beginning of July 1989 at the beginning of the third year). Based on student comments, extensive editing of the studies occurred before June 26, 1989.

\section{DISCUSSION}

Previous approaches to the use of computer based teaching of radiology to medical students have included computer screen text display with photographic image slide display, ${ }^{5}$ or low-cost, stand-alone, dedicated radiology systems with somewhat limited resolution and memory. ${ }^{6}$

We previously had used a videodisk-based system with a separate 525 -line monitor for text. Whereas this system has the advantage of demonstrating imaging studies in motion, such as real-time ultrasound, nuclear flow studies, digital subtraction angiography (DSA), and so forth, it had the following disadvantages in comparison to the currently used system: (1) lack of digital acquisition, storage, and its attendant reproducibility; (2) limited storage capacity; (3) inferior resolution in comparison with the current method; and (4) inability to postprocess local areas of the image. ${ }^{1}$

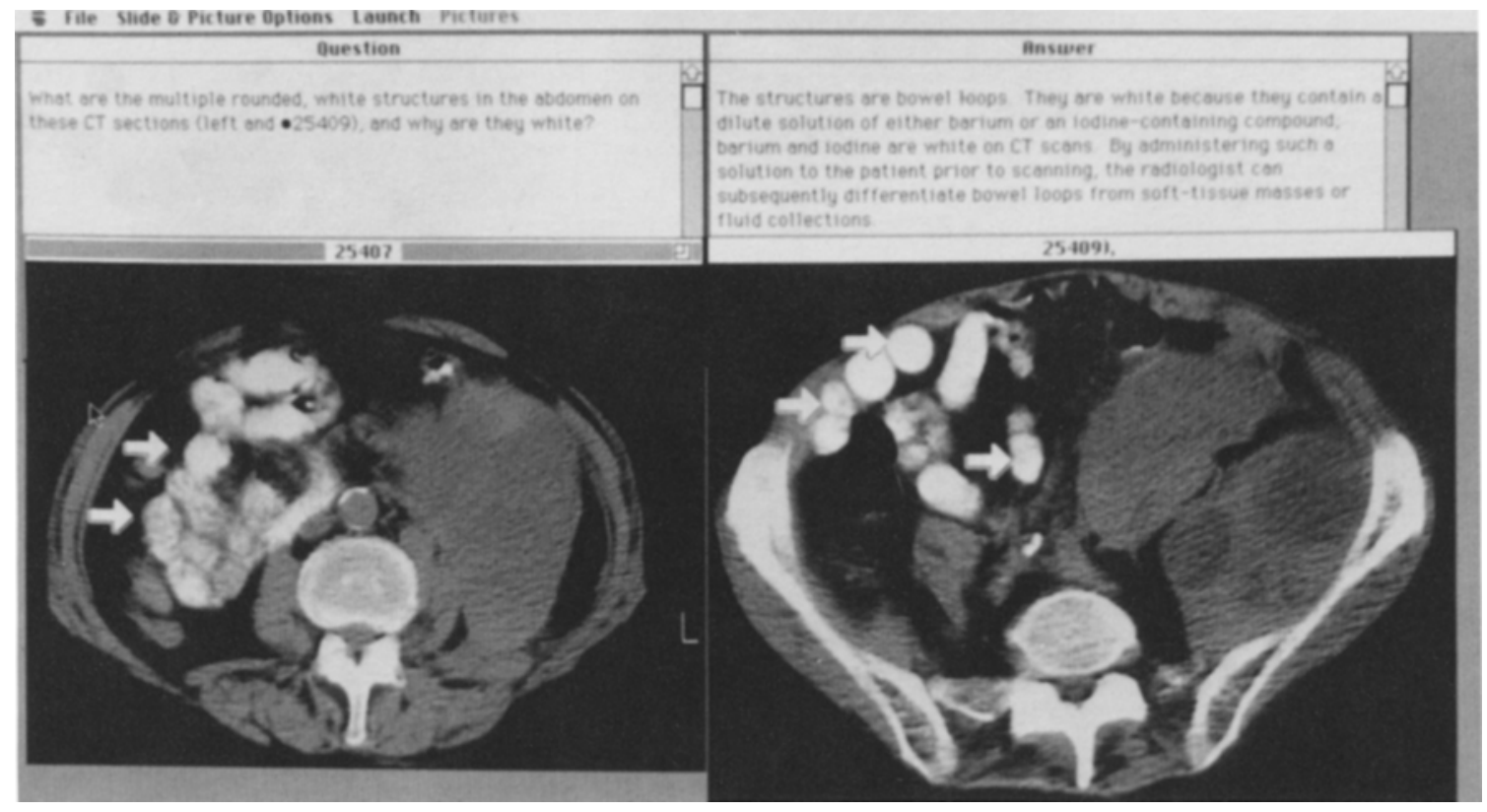

Fig 3. Multiple images are displayed with adequate resolution plus question-and-answer text. 


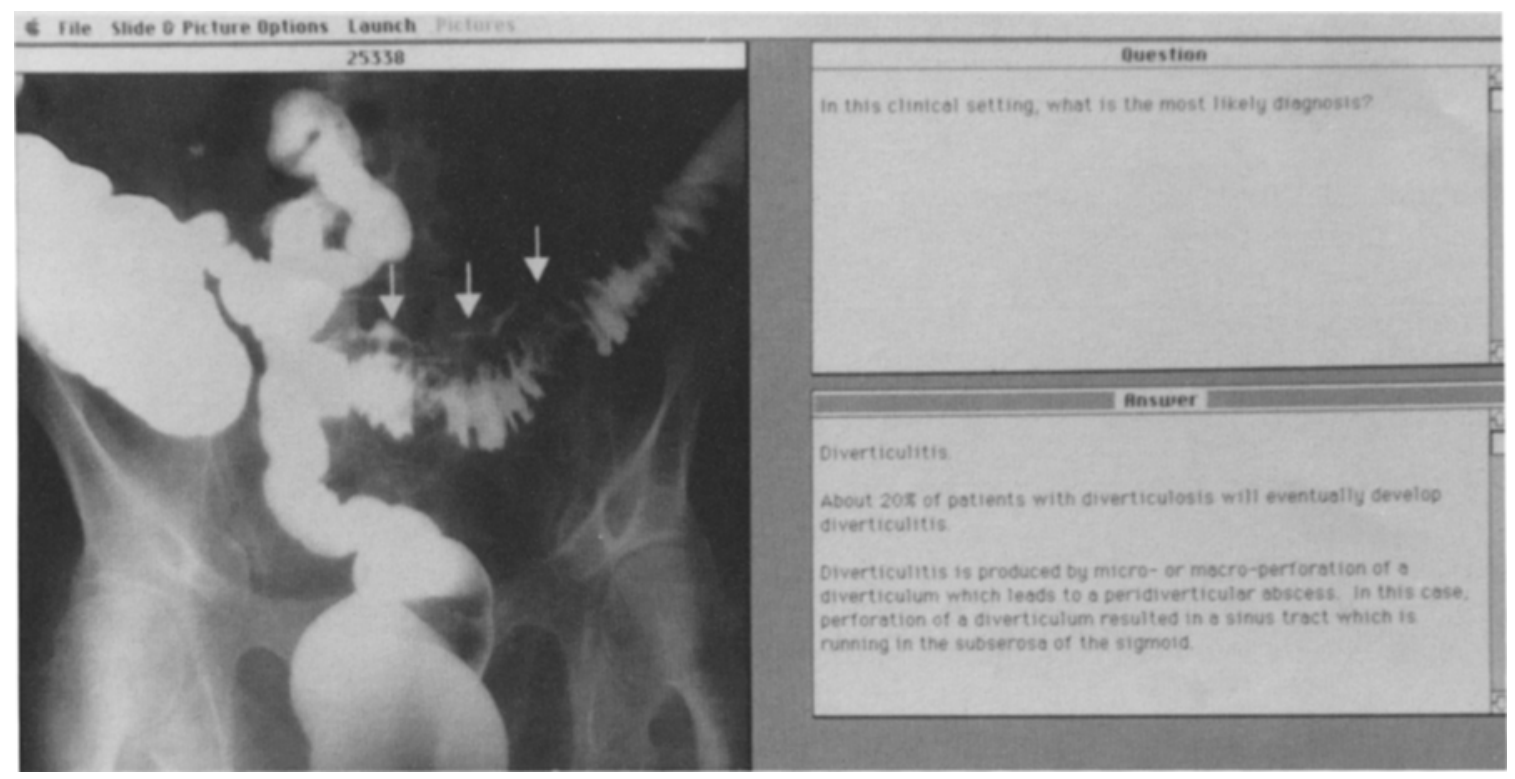

Fig 4. Five sets of questions and answers are related to image 25338. This is the third of five.

By using an existing high-resolution networked computer system used to teach a 6-month pathology course as well as physiology, neuroscience, biochemistry, parasitology, and microanatomy, we were able to take advantage of the fairly widespread availability of terminals in the medical school environment to teach the entire class of 104 students simultaneously. Beginning in late June 1989, the mandatory third-year Radiology Clerkship curriculum changed from a previous 2-week rotation of 12 to 13 medical students per group eight times per year to a single rotation of the entire class through radiology at the end of the second year. This required a major teaching effort by the radiology staff during the traditional vacation time of late June and July. In the previous course, each radiologist using radiographs or slides conducted a semipersonal tutorial to a relatively small group of medical students rotating through the department eight times per year. This was supplemented by a standard radiologic student text and a standard slidetape instruction series.

The current program (in use through 1991) is composed of (1) a 26-lecture series that is supplemented by a course syllabus that summarizes the lectures; (2) a standard medical student radiology text; (3) student rotations through all aspects of the radiology departments of the four affiliated hospitals in groups of four, where film-based tutorials are given by radiologist instructors (in addition to familiarizing the students with the hands-on aspects of various examinations); and (4) the above described computer-based instruction. The computerbased instruction parallels the lectures and other course material and is used between other teaching activities of the department as well as in the evenings. Students spend approximately 3 hours per day using RAD-MAC during the course. Because so many terminals are available, and because they are available at all hours of the day and night, the entire class may take advantage of this throughout the day. Groups of up to four students use each terminal. During the summer there appears to be no conflict in their use; however, if this course were given later during the academic year, competition for terminal use with students taking other subjects would probably occur.

Because the students had used essentially the same computer system in other prior courses, only minimal instruction on the operation for radiology was necessary.

In comparison with the slide-tape series, the initial group of students thought that the unedited, initially entered cases were not as clearly explained. However, the self-pacing aspect of the interactive computer system appeared to 
outweigh even this disadvantage. Subsequent editing, especially the placement of colored arrows on the radiologic findings when the answer was given in the text, improved the teaching value for medical students at the beginning of their clinical years.

A subsequent student feedback questionnaire used in the second year of this project after most of the editing was completed indicated that the students preferred the computer system to the lecture series or the course syllabus. However, student rotations through all aspects of the Radiology Department, and departments of the four affiliated hospitals, was still the most popular portion of the course.

After editing, on a scale of 1 to 4 students rated RAD-MAC image quality as 3.10 and the study material available in RAD-MAC as 3.24. Abdominal, gastrointestinal, and chest radiology were most frequently used. The most frequent complaint was the presence of too much text in the answers. Further revisions, adding more images, and reducing text will occur next year.

We have not yet conducted a trial comparing student learning during the lecture series versus learning using RAD-MAC. However, this year one of the second-year medical school courses will be offered as a lecture series versus computer instruction and the final examination results compared.

We plan to add additional terminals in the Radiology Department and expand the system to a radiology departmental teaching file with a $14 \times 17$-inch flatbed scanner and install it in at least two of our affiliated hospitals. One of these institutions, directly attached to the medical school, will be serviced by the Ethernet extension; the other, approximately 20 miles away, will be serviced by its own separate hard disc file.

Ultimately, we expect that our radiology computer-assisted teaching program will be integrated into a more comprehensive radiology teaching program involving a group of 11 medical schools, which recently founded the Northeastern Medical School Consortium for the purposes of exchanging and possibly integrating computer-based medical student teaching programs.

In conclusion, this high-resolution networked system provides availability of self-paced radiologic instruction to the entire medical student class simultaneously with adequate resolution for both radiologic imaging and considerable explanatory text on the same screen.

\section{REFERENCES}

1. Jaffe CC, Lynch PJ, Smeulders AW: Hypermedia techniques for diagnostic imaging instruction: Video disk echocardiography encyclopedia. Radiology 171:475-480, 1989

2. Aronberg DJ, Rodewald SS, Jost RG: Computerassisted instruction in radiology. Radiology 154:345-347, 1985

3. Squire LF: On teaching radiology to medical students: Challenges for the 90's. AJR 152:457-461, 1989
4. Squire LF, Novelline RA: Radiology should be a required part of the medical school curriculum. Radiology 156:243-244, 1985

5. Jacoby CG, Smith WL, Albanese MA: An evaluation of computer-assisted instruction in radiology. AJR 143:675677,1984

6. McGhee RB, Bennett WF, Morris CS, et al: Costeffective development of a computer-assisted instruction system. AJR 153:877-879, 1989 